\section{Revue de l'IFHA}

Revue de l'Institut français d'histoire en Allemagne

$6 \mid 2014$

IFHA 6

\title{
Atelier « Enseigner aujourd'hui la Première Guerre mondiale en France et en Allemagne : programmes, manuels et mémoires - Der Erste Weltkrieg im Unterricht in Deutschland und Frankreich : Lehrpläne, Schulbücher, Erinnerungskulturen »
}

Francfort-sur-le-Main, IFHA/Ziehenschule, 17 octobre 2014

\section{Pierre Monnet}

\section{OpenEdition}

Journals

Édition électronique

URL : http://journals.openedition.org/ifha/8006

DOI : $10.4000 /$ ifha. 8006

ISSN : 2198-8943

Éditeur

IFRA - Institut franco-allemand (sciences historiques et sociales)

Édition imprimée

Date de publication : 31 décembre 2014

ISSN : 2190-0078

Référence électronique

Pierre Monnet, « Atelier « Enseigner aujourd'hui la Première Guerre mondiale en France et en Allemagne : programmes, manuels et mémoires - Der Erste Weltkrieg im Unterricht in Deutschland und Frankreich : Lehrpläne, Schulbücher, Erinnerungskulturen » », Revue de l'IFHA [En ligne], 6 | 2014, mis en ligne le 31 décembre 2014, consulté le 19 avril 2019. URL : http://journals.openedition.org/ifha/8006 DOl : 10.4000/ifha. 8006

Ce document a été généré automatiquement le 19 avril 2019

CIFHA 


\section{Atelier « Enseigner aujourd'hui la Première Guerre mondiale en} France et en Allemagne : programmes, manuels et mémoires - Der Erste Weltkrieg im Unterricht in Deutschland und Frankreich : Lehrpläne, Schulbücher, Erinnerungskulturen »

Francfort-sur-le-Main, IFHA/Ziehenschule, 17 octobre 2014

Pierre Monnet

1 Depuis des mois, la commémoration du centenaire de la déclaration de la Première Guerre mondiale en 1914 place de nouveau au centre de l'attention la question des origines du conflit, de la participation des populations civiles à l'effort et aux destructions de guerre, de la culture de la violence qui s'installa au cœur des sociétés européennes, de l'état d'esprit des soldats, de la mobilisation des forces économiques et des ressources culturelles et symboliques au service d'un combat de longue durée.

2 Ces problématiques ne sont pas du seul ressort des spécialistes ou des historiens de métier, mais affectent aujourd'hui encore des couches très larges de la population par le biais des musées, par le truchement de la mémoire familiale et collective, par un grand nombre de publications, par la mise à disposition de documents sonores et visuels massivement digitalisés et par un vaste programme de commémorations publiques en Europe et dans le monde.

3 L'école, les enseignants, les élèves sont également concernés au premier plan par ce travail d'histoire et de mémoire, non seulement en 2014 même, mais également parce que 
la Première Guerre mondiale fait partie depuis longtemps et de manière diverse des programmes et des manuels scolaires. Ce sont justement la diversité de cette présence, la pédagogie de l'enseignement, la didactique documentaire, l'insertion de cet épisode dans un ensemble chronologique et spatial plus vaste qui ont constitué l'objet de la rencontre organisée conjointement par l'Institut Français d'Histoire en Allemagne et la Ziehenschule de Francfort. L'approche entendait privilégier un examen à la fois critique et pratique des programmes, des manuels et des mémoires sédimentées par l'institution scolaire dans une comparaison franco-allemande plongée dans une échelle européenne des manières d'enseigner, de transmettre et de raconter cet événement. Pour en discuter, des enseignants, des universitaires, des spécialistes de la didactique et de la pédagogie, des éditeurs de manuel, des concepteurs et responsables de programme se sont rencontrés une journée durant pour se livrer au double exercice de la présentation de thèses, d'hypothèses et d'interprétations et de la discussion au sein d'ateliers réunissant des enseignants qui, de surcroît, ont pu valider leur participation à cette journée au titre de la formation continue.

\section{ANNEXES}

Programme

GRUSSWORTE/MOTS D'ACCUEIL

Manfred EICHENAUER, Leiter der Ziehenschule Frankfurt am Main

Rolf KNIELING, Beauftragter der KMK für AbiBac, Hessisches Kultusministerium, Referat III.5: Europa, Internationales, Auslandsschulwesen, Fremdsprachen

Pierre MONNET, Directeur de l'Institut Français d'Histoire en Allemagne

09.30-13.00 Uhr/09 h 30-13 h 00

\section{IMPULSREFERATE/PRÉSENTATIONS}

Einleitung/Introduction : Prof. em. Dr. Etienne FRANçoIs (Berlin) : « Das fragmentierte Gedächtnis des Ersten Weltkrieges »

Dr. Vincent DUCLERT (MEN, EHESS Paris) : « Les programmes scolaires en France face à la Première Guerre mondiale »

Prof. Dr. Gerhard HENKE-BOCKSCHATZ (Universität Frankfurt/M.) : « Die Darstellung des Ersten Weltkriegs in aktuellen deutschen Schulgeschichtsbüchern zwischen Meistererzählung und Kompetenzentwicklung »

Diskussion/Discussion

Prof. Dr. Christine PFLÜGER (Universität Kassel) : « ‘Erster Weltkrieg’ im bilingualen Unterricht » 
Prof. Dr. Peter GEISS (Universität Bonn) : « Quellendigitalisate der Bibliothèque nationale als Chance und Herausforderung für den bilingualen Geschichtsunterricht : Julikrise und Erster Weltkrieg auf gallica.bnf.fr "

Diskussion/Discussion

Zusammenfassung/Conclusions : Prof. Dr. Pierre MONNET et Dr. Ralf-Olivier SCHWARZ

14.30-16.30 Uhr/14 h 30-16 h 30

\section{ATELIERS}

Atelier I : Lehrpläne, didaktische Konzepte und ihre Umsetzungen in deutschen und französischen Geschichtsbüchern

Moderation und Einleitung in das Thema/Animation et introduction : Dr. Rainer BENDICK (Osnabrück) : « Was ist guter Geschichtsunterricht über den Ersten Weltkrieg in Deutschland und Frankreich? - Deutungskonflikte und Deutungsansätze in deutschen und französischen Schulgeschichtsbüchern »

\section{Atelier II : Neuer Blick, neues Material : Der Erste Weltkrieg als Comic erzählt}

Moderation und Einleitung in das Thema/Animation et introduction : Julie CAZIER (Köln/ Cologne, Auteure de la bande dessinée franco-allemande « Carnets de bord 14-18: Quatre histoires de France et d'Allemagne "/Autorin des deutsch-französischen Comicheftes «Tagebuch 14-18 - Vier Geschichten aus Deutschland und Frankreich »

\section{Atelier III : Erinnerungen/Mémoires}

Moderation und Einleitung in das Thema/Animation et introduction : Xavier DE GLOWCZEWSKI (Lille) : « Pourquoi et comment enseigner la Première Guerre mondiale aujourd'hui en Europe ? Une approche comparée »

\section{AUTEUR}

PIERRE MONNET

(EHESS, Paris et IFHA, Francfort-sur-le-Main) 